\title{
La infancia anormal en Chile y la pedagogía experimental de Wilbelm Mann (1860-1920)
}

\author{
Gonzalo Salas; Hernán Scholten; Silvana Hernández-Ortiz; Claudio Rojas-Jara; \\ Ernesto L. Ravelo-Contreras
}

How to cite this article:

Salas, G., Scholten, H., Hernández-Ortiz, S., Rojas-Jara, C., \& Ravelo-Contreras, E.L. (2019). La infancia anormal en Chile y la pedagogía experimental de Wilhelm Mann (1860-1920). Acta Colombiana de Psicología, 22(2), $241-253$. doi: http://doi.org/10.14718/ACP.2019.22.2.12

Recibido, diciembre 15/2018; Concepto de evaluación, febrero 11/2019; Aceptado, febrero 26/2019

\author{
Gonzalo Salas* \\ Universidad Católica Del Maule, Talca, Chile \\ ORCID: https://orcid.org/0000-0003-0707-8188 \\ Hernán Scholten \\ Universidad de Buenos Aires, Buenos Aires, Argentina \\ ORCID: https://orcid.org/0000-0003-3366-2142 \\ Silvana Hernández-Ortiz \\ Universidad Católica del Maule, Talca, Chile \\ ORCID: https://orcid.org/0000-0001-8150-3416 \\ Claudio Rojas-Jara \\ Universidad Católica del Maule, Talca, Chile \\ ORCID: https://orcid.org/0000-0002-1698-6949 \\ Ernesto L. Ravelo-Contreras \\ Universidad Católica de Colombia, Bogotá, Colombia \\ ORCID: https://orcid.org/0000-0002-9565-0828
}

\begin{abstract}
Resumen
Los análisis realizados por Michel Foucault hacia mediados de la década de 1970 dan cuenta del surgimiento de un poder disciplinario en occidente y de la figura del "débil mental" en el ámbito educativo. Este panorama impulsa la aparición de diversas elaboraciones teóricas y modalidades de intervención denominadas por el autor como "función psi”, que en el caso de la infancia alcanza una notable productividad en la Francia de comienzos del siglo XX. El presente artículo retoma estas cuestiones con el fin de indagar, en el caso de Chile, el despliegue de esos saberes y prácticas psicológicas que se ocupan de aquellos niños que, por motivos diversos, no logran adaptarse a las normas que impone el dispositivo escolar. Se utilizó un método de tipo historiográfico, mediante el trabajo de archivo y uso de fuentes primarias con un complemento de fuentes secundarias. Entre los hallazgos, se analizan los cruciales aportes de Wilhelm Mann, pedagogo alemán y figura pionera de la psicología en Chile, respecto de la infancia anormal a comienzos del siglo XX. Con esto, se explora y analiza una parte de su producción para iluminar el modo en que Mann se introduce en los debates y los modelos institucionales europeos y comienza a pensar una modalidad de intervención sobre la infancia a nivel local.

Palabras clave: infancia anormal, saberes psi, pedagogía, Chile, Michel Foucault, Wilhelm Mann.
\end{abstract}

\footnotetext{
Departamento de Psicología, Facultad de Ciencias de la Salud, Universidad Católica del Maule, Talca, Chile. Tel.: +56712203398. gsalas@ucm.cl

Este artículo fue apoyado por una subvención de la Comisión Nacional de Investigación Científica y Tecnológica (CONICYT), a través del Fondo Nacional de Desarrollo Científico y Tecnológico, Proyecto FONDECYT N. ${ }^{\circ}$ 11150436, otorgado al autor Dr. Gonzalo Salas.
} 


\title{
Abnormal childbood in Chile and the experimental pedagogy of Wilbelm Mann (1860-1920)
}

\begin{abstract}
The analyzes carried out by Michel Foucault in the mid-1970s show the emergence of a disciplinary power in the West and the figure of the "mentally weak" in the educational field. This panorama drives the appearance of various theoretical elaborations and intervention modalities called by the author "psi function", which in the case of childhood reaches a remarkable productivity in France at the beginning of the 20th century. This article takes up these issues in order to investigate, in the case of Chile, the deployment of the psychological knowledge and practices that deal with those children who, for various reasons, fail to adapt to the rules imposed by the school system. A historiographic method was used, through the work of archiving and using primary sources with a complement of secondary sources. Among the findings, the crucial contributions of Wilhelm Mann, a German pedagogue and a pioneer figure of psychology in Chile regarding abnormal childhood at the beginning of the 20th century are analyzed. Based on this, a part of his production is explored and analyzed to illuminate the way in which Mann introduces himself into European institutional debates and models and begins to think about an intervention modality on children at the local level.

Key words: abnormal childhood, psi knowledge, pedagogy, Chile, Michel Foucault, Wilhelm Mann.
\end{abstract}

\section{A infância anormal no Chile e a pedagogia experimental de Wilhelm Mann (1860-1920)}

Resumo

\begin{abstract}
As análises realizadas por Michel Foucault até meados da década de 1970 tratam do surgimento de um poder disciplinar no ocidente e da figura do "débil mental" no contexto educativo. Esse panorama impulsiona a aparição de diversas elaborações teóricas e modalidades de intervenção denominadas pelo autor como "função psi”, que, no caso da infância, alcança uma notável produtividade na França do início do século XX. O presente artigo retoma essas questões com o objetivo de indagar, no caso do Chile, o desdobramento desses saberes e práticas psicológicas que se ocupam daquelas crianças que, por diferentes motivos, não conseguem se adaptar às normas impostas pelo dispositivo escolar. Utilizou-se um método de tipo historiográfico, por meio do trabalho de arquivo e uso de fontes primárias com um complemento de fontes secundárias. Entre as descobertas, analisam-se as colaborações cruciais de Wilhelm Mann, pedagogo alemão e figura pioneira da psicologia no Chile, com respeito à infância anormal no início do século XX. Com isso, uma parte de sua produção é explorada e analisada para iluminar o modo em que Mann se introduz nos debates e nos modelos institucionais europeus e começa a pensar em uma modalidade de intervenção sobre a infância em um contexto local.

Palavras-chave: infância anormal, saberes psi, pedagogia, Chile, Michel Foucault, Wilhelm Mann.
\end{abstract}

\section{Introducción}

A mediados del siglo XIX inició un período fundamental en la organización de la educación en Chile-especialmente en lo que se refiere a la instrucción primaria- que se extendió hasta comienzos del siglo XX (Cox \& Gysling, 2009; Egaña, 2000; Labarca, 1939; Muñoz, 1918; Núñez, 1883; Serrano, Ponce de León \& Rengifo, 2012). En efecto, a partir de la Ley Jeneral de Instrucción Primaria de 1860 (Larraín \& Larraín, 1871), comenzó una paulatina institucionalización y regulación estatal de la educación que terminó de definirse hacia 1920 con la ley 3.654 de Educación Primaria Obligatoria (Dirección Jeneral de Educación Primaria, 1920), la cual instauró un proceso complejo, cuyo trayecto no parece seguir un movimiento progresivo, uniforme o ascendente. Es en este contexto que el presente artículo busca indagar sobre los saberes y prácticas psicológicas que surgieron a partir de los residuos que produce la disciplina escolar, es decir, de aquellos niños que no logran -de diversos modos-adaptarse a las normas que impone la escuela.

El ejemplar más ilustrativo de estos residuos escolares es el constructo de "débil mental" (Foucault, 2005), aspecto que el lenguaje kantiano tempranamente ha descrito, evidenciando un discurso despectivo y segregador, como la tónica de las clasificaciones médicas del siglo XX en los manuales de psiquiatría contemporáneos (p. ej., Kant, 1764/2018; Lapoujade, 2015; Lieberman, 2016).

En este sentido, es importante referir el surgimiento de la anormalidad infantil en la Europa del siglo XIX -principalmente en Francia- en relación con la clasificación de los idiotas e imbéciles por parte de la psiquiatría de la época (Huertas, 2011, 2014), ya que es a la luz de estos debates y problemáticas que surge el tránsito de estas personas 
hacia el mundo educativo, en el cual se van desplegando las primeras técnicas que permitieron su detección, exclusión y reubicación en diversas instituciones.

Teniendo esto en cuenta, en el presente artículo se utiliza una serie de conceptos propuestos por el filósofo Michel Foucault con la intención de disponer de un marco teórico que sirva de referencia para analizar la infancia anormal y la pedagogía experimental de Wilhelm Mann aplicada desde principios del siglo XX en el Instituto Pedagógico de la Universidad de Chile, con un alto impacto para la educación nacional (Parra, 2015; Salas \& Lizama, 2013). Con esto, se intenta mostrar que dicho pedagogo alemán, figura pionera de la psicología chilena, fue uno de las primeros en ocuparse del problema de la infancia anormal a nivel local.

De este modo, el objetivo del presente trabajo fue analizar una parte de su producción, sobre todo los trabajos publicados entre 1905 y 1915 (Mann, 1905a, 1905b, 1906, 1908, 1909, 1910, 1912, 1915a, 1915b), donde se encuentran las primeras referencias a la problemática de los niños anormales - principalmente, los débiles y retrasados mentales- como foco de preocupación para la pedagogía en Chile, destacando el modo en que Mann se introduce en los debates y los modelos institucionales que se instalaron en Europa para comenzar a pensar una modalidad de intervención sobre la infancia.

Este trabajo sigue el método historiográfico (Carr, 1993; De Mussy \& Valderrama, 2010; Klappenbach, 2014; Rosa, Huertas \& Blanco, 1996), mediante el uso de fuentes primarias - relevadas con una rigurosa búsqueda realizada en diversos archivos- obtenidas principalmente en el Salón de Investigadores de la Biblioteca Nacional de Chile. A dichas fuentes debe sumarse el complemento de fuentes secundarias, lo que permite una revisión, reconstrucción y análisis exhaustivo del objeto de estudio.

\section{El surgimiento del retraso mental como residuo de la disciplina escolar y objeto de la "función psi"}

Entre noviembre de 1973 y enero de 1974, Foucault presentó en el Colegio de Francia su curso sobre El poder psiquiátrico (Foucault, 2005), en el cual se retomaron y ampliaron algunas temáticas que el autor había presentado en cursos anteriores, como La sociedad punitiva (Foucault, 2016) y La verdad y las formas jurídicas (Foucault, 2017). Entre ellas, destaca la cuestión del poder disciplinario -o simplemente, la disciplina- abordado por primera vez en la clase del 14 de marzo de 1973 (Foucault, 2016).

Al respecto, Foucault propone explorar la hipótesis sobre la existencia de un poder disciplinario en la sociedad
(Foucault, 1999, 2005), que se distingue de otro tipo de poder que le antecede históricamente, denominado poder soberano. Para mostrar las características, diferencias y relaciones entre ambos conceptos, el autor emprende una indagación histórica que, en lo esencial, se remonta a los siglos XVIII y XIX, con un especial interés en destacar una serie de transformaciones que tienen lugar en diferentes espacios de la sociedad francesa de la época con el fin de dar cuenta de su disciplinarización y señalar cuatro características principales de este mecanismo de poder.

La primera característica de la disciplinarización es la captura total y exhaustiva del comportamiento del individuo, del cuerpo y del tiempo en su totalidad, a través de, por ejemplo, la disciplina militar y el acuartelamiento -que apareció desde fines del siglo XVII-. En el caso de la escuela, es posible apreciar la imposición a los niños del espacio aúlico y de una carga horaria, que regula gran parte de su vida diaria (Foucault, 2005).

La segunda característica hace referencia a que el poder disciplinario implica un carácter panóptico, en tanto se está perpetuamente en situación de ser observado (Foucault, 2005). En este sentido, la disciplina mira hacia el futuro, hacia un estadio en el que "todo funcione por sí solo", en el que la propia vigilancia sea innecesaria o apenas virtual. Como garantías de ese tipo de "captura permanente y global" de la disciplina, Foucault menciona, además del ejercicio, la escritura, el registro y la anotación de lo que hace y dice el individuo, lo que más tarde es esquematizado y centralizado en dispositivos de control, tales como el boletín de calificaciones, el prontuario, el legajo o la hoja clínica, etc. (Foucault, 2005). Este control y visibilidad permanente de los cuerpos propios le provee a la disciplina la posibilidad de una rápida ejecución de dominio y poder. De hecho, lo propio de la disciplina sería tender a intervenir incluso antes de que el acto se produzca:

Aun antes de que el gesto sea una realidad debe poder identificarse algo, y el poder disciplinario debe intervenir: intervenir, en cierto modo, antes de que la manifestación misma del comportamiento, antes del cuerpo, el gesto o el discurso, en el plano de la virtualidad, de la disposición, la voluntad, al nivel del alma $[\ldots]$, un alma muy diferente de la que habían definido la práctica y la teoría cristiana (Foucault, 2005, p. 73).

En una tercera característica, se menciona que los dispositivos disciplinarios son isotópicos (Foucault, 2005), lo cual implica que no dejan lugar para el azar o, al menos, que tiende a reducir el azar a su mínima expresión, y que cada uno de sus componentes tiene una posición precisa respecto a los demás, determinada por una estricta jerarquía. Aquí, Foucault menciona como ejemplo el modelo escolar de los 
jesuitas y de los Hermanos de la Vida Común, en el cual el lugar que los individuos ocupaban en la clase dependía de sus resultados escolares: los más exitosos ocupaban los primeros asientos, los más cercanos al profesor, y su ubicación se alejaba a medida que se aproximaban al fracaso escolar (Foucault, 1990, 2005). Adicionalmente, esta isotopía, incluso, implica una articulación entre diferentes dispositivos disciplinarios, como la escuela, el hospital, el cuartel, etc., en donde existe la posibilidad de que las personas transiten de uno a otro (Foucault, 1990, 2005). De hecho, acá la escuela funciona como un dispositivo que prepara el pasaje de los sujetos a los demás sistemas disciplinarios.

Finalmente, como cuarta característica se menciona que la isotopía disciplinaria implica siempre la producción de residuos, de aquello que queda por fuera, que escapa de la disciplina, que choca con ella. Así, además del desertor, que solamente existe a partir de la constitución de un ejército disciplinado, Foucault menciona el caso del débil mental en la escuela y del loco, que se convierten en los residuos de todos los espacios disciplinarios (Foucault, 2001, 2005, 2014a).

Estos residuos, que no son un mero accidente u obstáculo, sino un efecto propio de la disciplina, dan lugar a nuevos espacios disciplinarios, cuyo propósito es normalizarlos; tal es el caso de las escuelas especiales destinadas a los débiles mentales.

Ahora bien, separándose de este dispositivo disciplinario, es entonces cuando Foucault referencia a la familia, no para caracterizarla como parte de la disciplinarización, sino para mostrar que -hacia el siglo XIX-se constituye como una célula, un resto de esa modalidad de poder previa y casi opuesto a la disciplina: el poder soberano.

Pese a esta diferencia entre los dispositivos disciplinares y la familia, esta última cumple una función esencial en el marco de la sociedad disciplinaria que se constituye en el siglo XIX. En efecto, esta familia fue redefinida e intensificada, pero limitada a su formato nuclear: la pareja parental y sus hijos (Goody, 2009; Smadja, 2013); "es la instancia de coacción que va a fijar de manera permanente a los individuos a los aparatos disciplinarios, que en cierto modo va a inyectarlos en ellos" (Foucault, 2005, p. 105). La imposición de la educación primaria obligatoria solo fue posible gracias a la intervención de la soberanía familiar.

Incluso, la familia cumplía una función adicional como punto de enganche, en la articulación entre diferentes poderes disciplinarios, como instancia social que garantiza el pasaje de un dispositivo disciplinario a otro: de la escuela al taller, al cuartel, a la cárcel o al manicomio. Una prueba de esta segunda función de la familia en las sociedades disciplinarias es que, cuando un individuo es rechazado por algún sistema disciplinario, es enviado de regreso con su familia (Foucault, 2005); y, de hecho, se convoca o demanda a la familia cuando un niño comienza a dar muestras de ser un potencial residuo de la disciplina escolar.

Posteriormente, Foucault constató la aparición, hacia mediados del siglo XIX, de dispositivos disciplinarios nuevos, como las casas para niños expósitos, los orfelinatos, las escuelas para niños pobres, la escolarización del niño obrero y los hogares para delincuentes juveniles, a partir de lo cual surge el concepto de la infancia en peligro (Foucault, 2005; Larrosa, 1995). A raíz de esto, emergió entonces la denominada "función psi", utilizada por psicólogos, psiquiatras, psicoanalistas, criminólogos, etc., "agentes de la organización de un dispositivo disciplinario que va a ponerse en marcha [...] cuando se produzca un vacío de la soberanía familiar" (Foucault, 2005, p. 110).

Así, la psiquiatría surgió como respuesta a la soberanía familiar incapaz de contener al individuo, que más tarde se fue extendiendo a los demás dispositivos disciplinarios. Para inicios del siglo XX, la "función psi" llegó a convertirse "en el discurso y el control de todos los sistemas disciplinarios [...] es el discurso y la introducción de todos los esquemas de individualización, normalización y sujeción de los individuos dentro de los sistemas disciplinarios" (Foucault, 2005, p. 111), que tienen como referencia constante a la familia: el surgimiento de la psicopedagogía y la psicología educativa, el uso de evaluaciones psicológicas para legitimar las decisiones escolares -como la segregación escolar en relación a patologías del aprendizaje-, y psicometrías de la inteligencia que establecieron una relación entre las prácticas educativas y las psicológicas, las cuales, complementadas por medio de estos nuevos saberes psicologizantes, son ejemplos -a nivel de los saberes y discursos- de esa "función psi" al interior de la disciplina escolar (Parra, 2015; Terigi, 2009).

Adicionalmente, Foucault (2005) propuso que la generalización del poder psiquiátrico hacia los establecimientos educativos y sanitarios tuvo como referente principal la figura del niño, y, para dar cuenta de esta hipótesis, se ocupa de exponer un doble proceso: por un lado, la elaboración teórica de la noción de imbécil o idiota como fenómenos que no pertenecen al ámbito de la locura; y por otro, la introducción de la idiotez en el espacio psiquiátrico manicomial.

Así, hasta finales del siglo XVIII, la imbecilidad, estupidez o idiotez eran consideradas por la psiquiatría como un tipo de locura, una forma particular de la serie de la demencia (opuestas a las que tienen la forma del furor), donde el delirio llega a su punto más agudo y generalizado; pero hacia mediados del siglo XIX se desarrolla una 
reelaboración de estas categorías (Noll \& Trent, 2004; Postel \& Quétel, 2000).

De este modo, aunque menciona a otros autores -como Esquirol y Belhomme-, Foucault se detiene en el Traitement moral, hygiène et éducation des idiots et des autres enfants arriérés [Tratamiento moral, higiene y educación de idiotas y otros niños retrasados] de Seguin (1846), que "propone los conceptos fundamentales de la psicología y la psicopatología del retraso mental que se desarrollarán a lo largo del siglo XIX" (Foucault, 2005, p. 239), donde le interesa destacar la distinción que realiza este autor entre idiotas y retrasados, entre aquellos en que el desarrollo se interrumpió y aquellos en que el desarrollo es más lento. Acá, el desarrollo se comprende como un proceso temporal que afecta a todos los individuos, y puede ser considerado como una norma o, más bien, desde una doble normatividad: en el caso del idiota respecto al adulto (como punto culmine) y en el retraso mental respecto a los otros niños.

Por lo tanto, estas dos, especialmente el retraso mental, no consisten en enfermedades, sino en tipos de niñez y variedades dentro del desarrollo normativo infantil: no son niños enfermos, sino que, en el caso del retrasado mental, se trata de un niño anormal (Foucault, 2001, 2005) que, por tanto, no debe ser tratado como un enfermo, sino como un niño, y su cura consistirá en imponerles un abordaje psicoeducativo (Foucault, 2005).

A diferencia de la enfermedad, la anomalía no genera síntomas, sino que tendría, como fenómeno positivo, la liberación del instinto, que es algo propio de la infancia, que está presente desde el comienzo y que aparece claramente en la idiotez o el retraso mental (Foucault, 2005).

No obstante, al mismo tiempo que se introducía esta distinción, se produjo la incorporación de la idiotez en el espacio psiquiátrico; en principio, aclara Foucault, en instituciones de sordomudos. Esto no fue efecto de las regulaciones de la enseñanza primaria que tuvieron lugar en Francia en 1833 (Ravier, 2012), sino que la cuestión que se planteaba era dónde ubicar a estos niños en función del trabajo de los padres, y es en este sentido que puede relacionarse con la apertura de salas de asilos, guarderías, jardines de infantes y con la escolarización de los niños. Teniendo esto en cuenta, en 1834 se fundó el Instituto de Ortofrenia para el tratamiento de niños pobres con deficiencia mental (Voisin, 1843), con el fin de ubicarlos, más tarde, en pabellones dentro de los hospicios que estaban destinados a los débiles mentales, idiotas y, a menudo, histéricas y epilépticos -que en todos los casos eran considerados como niños-.

Ahora bien, Foucault no incursionó en posteriores cursos, ni siquiera en el que dedica a Los anormales (Foucault, 2001), en los avatares de la infancia anormal durante las décadas inmediatamente posteriores, y no aborda una etapa que podría considerarse fundamental en relación con la temática que aquí interesa abordar y que transcurre, en lo esencial, hacia comienzos del siglo XX.

En este contexto, hacia finales del siglo XIX se fundó la Unión Francesa para el Salvataje de la Infancia (1887) y, tres años más tarde, el Patronato de la Infancia y de la Adolescencia, cuyo fin era educar y ubicar a los niños desviados, vagos o delincuentes juveniles de 8 a 18 años de edad, $\mathrm{y}$ también se promulgaron las leyes sobre la privación paterna -en 1889- y sobre la instrucción obligatoria de los delitos cometidos por menores -en 1890-(Muel-Dreyfus, 1975).

Adicionalmente, en este momento aparece la figura de Alfred Binet, fundador de la revista L'Année psychologique -en 1894- y que, a partir de los años siguientes, ya a comienzos del siglo XX, asume la dirección del Laboratorio de Psicología de la Sorbona (Binet, 1906). Particularmente, en 1904 participó de una comisión interministerial, dirigida por Léon Bourgeois, que reunía a educadores, médicos, hombres de ciencia y representantes de los servicios administrativos interesados para estudiar de las condiciones bajo las cuales debe proporcionarse la educación de niños anormales (Binet \& Simon, 1907); y, en el marco de esa investigación, llevó a cabo una serie de trabajos con Théodore Simon -publicados en L'Année psychologique-, donde propuso "un método para medir la inteligencia basado en la aplicación de una escala de tareas ordenadas por su dificultad creciente de acuerdo con la edad de los niños" (Huertas \& del Cura, 1996, p. 117). Como se verá más adelante, este método fue ampliamente aceptado en numerosos países, y diversos investigadores comenzaron a producir las versiones locales de lo que comenzaba a conocerse como el test de inteligencia de Binet-Simon (García, 2016; García-Yagüe, 2010; Salas, 2012; Sánchez \& Valderrama-Iturbe, 2001).

Con esto, en dicho período se propuso añadir dos nuevas categorías al sistema de clasificación de los $n i$ ños anormales formulados por la psiquiatría hasta ese momento (idiotas e imbéciles): los retrasados y los inestables (Muel-Dreyfus, 1975). Para Binet, los retrasados no presentaban una anomalía bien definida del carácter y no aprovechaban o aprovechaban muy poco la enseñanza dada comúnmente con los métodos habituales, mientras que los inestables -término médico que corresponde a la expresión más escolar de indisciplinado- eran principalmente anormales del carácter (Binet \& Simon, 1904, 1907, 1908). No obstante, una distinción aún más relevante es la que realizó este autor entre los anormales de hospicio y los anormales de escuela, la cual apuntaba principalmente a señalar el tipo de anormales que debían ser admitidos en el ámbito escolar, es decir, quienes serían capaces de llegar a ganarse parcialmente la vida (Binet \& Simon, 1907). Esta 
distinción dejó fuera del ámbito pedagógico a los idiotas, pero hizo ingresar a los imbéciles y, con mayor razón, a los débiles mentales.

Como cierre de este período puede considerarse la promulgación en Francia de la ley sobre la creación de clases de perfeccionamiento anexas a las escuelas elementales públicas y de escuelas autónomas de perfeccionamiento para niños retrasados, erradicada el 15 de abril de 1909 (Huertas, 2008).

Ahora bien, tras la presentación de este panorama sobre los residuos en la disciplina escolar y la "función psi" en Francia, a continuación interesa explorar sus características en Chile. Para esto, es preciso detenerse en la modalidad en que se implementa allí la escolarización de la infancia.

\section{El "Estado docente" y la escolarización del niño chileno hacia mediados del siglo XIX}

¿De qué modo se organiza la educación en Chile en el proceso de transformación del país en un Estado moderno? Sin ánimo de exhaustividad, se podría comenzar señalando que, hacia mediados del siglo XIX, había solo 56 escuelas fiscales en Chile, ninguna de ellas ubicada en su capital (Labarca, 1939). Por el contrario, en ella funcionaban numerosas instituciones conventuales, particulares o municipales, cada una con un estipendio particular. Además, el porcentaje de población escolarizada era muy bajo y ascendía apenas al $1 \%$ de la población (Labarca, 1939).

La tarea de imponer un sistema de educación primaria gratuito a nivel nacional fue, sin duda, el producto de un proceso atravesado por proyectos diversos y polémicos, y sus bases se remontan a la década de 1840, durante la presidencia de Manuel Bulnes, en la que, por iniciativa de Manuel Montt, en su rol de Ministro de Justicia e Instrucción Pública, se creó la Escuela Normal de Preceptores (Serrano et al., 2012). En esta, se le adjudicó un particular valor a la figura de su director, el intelectual argentino, Domingo Faustino Sarmiento, quien se ocupó de dictar las clases de las diferentes asignaturas del plan de estudios de esa institución.

En ese marco, Sarmiento incorporó las experiencias que recogió en sus viajes por comisión del Gobierno a España, Francia, Italia, Alemania e Inglaterra, y, posteriormente, a Canadá y Estados Unidos, para regresar a Chile en 1848 (Labarca, 1939), y, con base en el informe que presentó al gobierno chileno, al año siguiente se publicó la primera edición de Educación Popular (Sarmiento, 1896a). Las propuestas formuladas por Sarmiento en este volumen influyeron significativamente en los debates sobre la educación en Chile durante esos años, especialmente en los proyectos de ley que Antonio García Reyes y Manuel Montt presentaron poco después (Tedesco \& Zacarías, 2011).

Para Sarmiento, de acuerdo con lo expuesto en la introducción de Educación popular, la educación de las masas, debido a su impacto sobre la moral y la inteligencia de los hombres, permitía elevar la dignidad del Estado. Además, desde una perspectiva preventiva, Sarmiento resalta que el nivel del cultivo de la razón y de los sentimientos morales de las masas es directamente proporcional a su respeto por la vida y la propiedad. Por esto, se vuelve evidente entonces que la educación era considerada una herramienta esencialmente civilizatoria, normalizadora, que lograba institucionalizar la vida social en pos del bien del Estado y la identidad nacional (Serrano et al., 2012).

En este sentido, resulta pertinente hacer referencia aquí a la Sociedad de Instrucción Primaria de Santiago, fundada en junio de 1856 por un grupo de jóvenes intelectuales, que incluía a Miguel Luis Amunátegui, cuyo principal objetivo era combatir la ignorancia, "que impide el desarrollo rápido y completo de la civilización en Chile" (Labarca, 1939, p. 141). De este modo, retomaban el título de una de las más prestigiosas obras de Sarmiento: Civilización o barbarie (Sarmiento, 1896b), con lo que se perfila la escuela como mecanismo civilizatorio que, más allá de transmitir ciertos saberes o contenidos, o incluso a través de ellos, busca introducir modificaciones y transformaciones que apuntan a una civilización de los comportamientos (Salas, Scholten \& Rey-Anacona, 2015).

Desde la perspectiva de un Estado nacional moderno, la escuela tiene un papel fundamental que cumplir en la construcción de una cultura compartida básica que permita cohesionar a sus habitantes, construir un pasado y proyectar un futuro. Estos lineamientos y estándares educativos facilitaron el objetivo gubernamental de trasfondo: generar una unidad nacional a través de valores, cultura e idiosincrasia en común (Parra, 2015). Por esto resultaba principalmente impulsada y sostenida por las autoridades estatales, y no tanto desde las demandas del ámbito laboral o de familia de las clases populares, los cuales llegaron a considerar a la escuela más como un obstáculo que como un aliciente (Egaña, 2000).

A esto se lo plantea, incluso, en términos de una contienda histórica entre la escuela y la calle, esta última entendida como sinónimo de barbarie y como un espacio libre de normas, autoridades, horarios y hábitos (Illanes, 1991). En muchos casos, se luchaba contra las costumbres cotidianas de la comunidad, hasta con la disposición del espacio y el tiempo del dispositivo escolar para convertirse en los preceptores de agentes civilizatorios que debían ser un modelo, incluso desde las cuestiones más básicas, relacionadas con el aseo y la vestimenta. 
Es así como el Estado docente y la escolarización de los niños, respaldados por los saberes "psi" dentro de la pedagogía, establecieron la existencia de sistemas de evaluación y clasificación con base en sus capacidades cognitivas, y con esto se desprendieron procesos de selección, normalización, jerarquización y centralización, constituidos por un poder disciplinario, donde la ciencia -en este caso, la psicología y la pedagogía-, realizaron, tal como afirmaba Foucault (2014b), una disciplinarización de los saberes.

Finalmente, el 24 de noviembre de 1860, hacia el final del segundo período presidencial de Manuel Montt, el Congreso Nacional de Chile promulgó la Lei Jeneral de Instrucción Primaria (Campos, 1960; Larraín \& Larraín, 1871), que buscaba brindar cierta base al sistema educativo nacional, además de atribuir al Estado la responsabilidad respecto de la educación elemental y establecer su gratuidad y la inclusión de ambos sexos.

Ahora bien, uno de los principales problemas a los que se enfrentó el sistema escolar chileno durante varias décadas siguió siendo la baja matricula y, eventualmente, la deserción escolar (Rojas-Flores, 2010). En efecto, la disciplina escolar no llegaba a imponerse de manera clara y contundente, muchos no llegaron jamás a ingresar a la institución e, incluso, algunos desertaban o eran retirados por sus padres para cooperar en la economía familiar: estos no podían ser considerados sus residuos, sino que pasaban a formar parte de los incivilizados que quedaban por fuera de su campo de acción. En el caso de los niños, estos llamaban principalmente la atención por su miserable situación económica, que no solamente les impedía adquirir los útiles escolares (pluma, cuadernos, libros), sino, incluso, una vestimenta decente.

Por otra parte, las observaciones sobre el comportamiento y la actitud de los escolares pocas veces refieren a la insolencia o la mala conducta, sin embargo, en estos casos, las medidas que se tomaban eran principalmente represivas: el castigo corporal era un recurso común, aunque fue cada vez más condenado (Egaña, 2000). A medida que el fin de siglo se fue acercando, se desarrolló una mayor sensibilidad hacia los castigos corporales, especialmente en aquellos casos que demostraban una desmedida crueldad. Así, el Reglamento para la enseñanza i régimen interno de las escuelas elementales, de 1883 (Ministerio de Justicia, Culto e Instrucción Primaria, 1833), aunque no prohibió explícitamente los castigos corporales, señaló como castigos la reconvención, la privación de recreo, la inscripción en el cuadro reprobativo, la comunicación a los padres $\mathrm{y}$, eventualmente, la expulsión, como de hecho se pudo apreciar en el caso de la Escuela Normal (Labarca, 1939).

Por otra parte, el primer Congreso Pedagógico, celebrado en Santiago, en 1889 (Núñez, 1890), se presentó como un espacio de polémicas sobre las prácticas educativas escolares que permitió reconsiderar el valor científico de la pedagogía. Dos miembros de la junta directiva de ese evento se destacaban como intensos activistas por la educación: Claudio Matte, presidente de la Sociedad de Instrucción Primaria desde 1892, quien mostró una marcada preocupación por cómo y qué se enseñaba y se aprendía, autor del silabario que, desde 1894 y durante muchas décadas fue literatura privilegiada para la enseñanza de la lectoescritura en Chile; y José Abelardo Nuñez, quien llegó a ser Inspector General de Instrucción Primaria, y compartía con Matte una verdadera fascinación con las orientaciones pedagógicas que surgían en Europa, especialmente en Alemania.

En esa misma época, el modelo francés que se había impuesto hasta ese momento en la formación de maestros fue abandonado en pos del modelo alemán, el cual se mantuvo unos veinte años (Salas, 2012; Salas, Norambuena, Scholten \& Torres-Fernández, 2018), con lo que se inició lo que se denomina, retomando la expresión de Collier y Sater, el "embrujamiento alemán" (De la Barra, 1899). Con esto coincide Amanda Labarca, debido a que señala el contrapeso que la cultura alemana comienza a presentar ante la influencia francesa que se imponía hasta la década de 1880; impacto que no se limita al ámbito pedagógico, con la llegada a Chile de profesores provenientes de Alemania a las escuelas normales chilenas (Caiceo, 2011), sino que llegó a extenderse hasta el propio ejército local.

Adicionalmente, durante la presidencia de José Manuel Balmaceda (1886-1891) se inició un amplio plan de mejoramiento de la educación que incluyó la creación del Instituto Pedagógico de la Universidad de Chile, en el año 1889 (Ciudad, 1989; Fuentealba, 1964; Mellafe, 1988; Mellafe \& González, 2007), institución que tenía la misión de formar docentes para las distintas especialidades que se impartirían en los liceos y para su primer cuerpo académico, conformado por una aplastante mayoría de profesores alemanes contratados por el Estado. Entre estos profesores se encontraba el Dr. Georg Heinrich Schneider, a quien se le encargó dictar las cátedras de Filosofía y Pedagogía. Es en este marco que resulta posible introducir los aportes de Wilhelm Mann, con los cuales se podrá delinear el abordaje inicial del problema de los residuos de la disciplina escolar a nivel local.

\section{Las primeras referencias de la psicología a la anormalidad infantil en Chile: la pedagogía experimental de Wilhelm Mann}

La figura de Wilhelm Mann ha sido objeto de análisis de numerosas investigaciones históricas que se ocupan de ubicarlo como una figura pionera de la psicología en 
Chile (Barrera, 2010; Salas \& Lizama, 2013; Urzúa, VeraVillarroel, Zúñiga \& Salas, 2015). Nacido en la ciudad de Bielefeld, Alemania, en 1874, realizó su formación en Filosofía en la Universidad de Frankfurt y llegó a Chile en 1903 para dirigir la cátedra de Pedagogía del Instituto Pedagógico de la Universidad de Chile, cargo que había dejado vacante su compatriota Georg Heinrich Schneider, al jubilarse (Letelier, 1940). Hacia 1905 se publican, en los Anales de la Universidad de Chile, los primeros textos que Mann dedica a la temática de la pedagogía experimental (Mann, 1905a, 1905b, 1906), y en adelante, la mayor parte de su producción escrita estuvo dedicada a tópicos que conectaban los avatares del ámbito educativo con los saberes y prácticas psicológicas (Mann, 1908, 1909, 1912, 1915a).

En octubre de 1906, Mann inició un viaje en el que, durante seis meses, visitó varias ciudades de Europa y de Estados Unidos, además de Buenos Aires, para observar la organización de diversos laboratorios y seleccionar los instrumentos que resultaran óptimos para la instalación de un gabinete en Chile. Al regresar a Santiago, redactó un extenso informe que fue publicado en dos partes (Mann, 1908, 1909), e instaló, en el Instituto Pedagógico, un laboratorio de psicología, el segundo en el país (Salas \& Lizama, 2013) tras la fundación del Laboratorio de Psicología de la Escuela Normal de Copiapó (Ramírez \& Navarrete, 1932).

De los textos que publicó - bajo diversas denominacionesdurante su estadía en Chile, Mann se ocupó del problema de la infancia anormal, y entre estos destaca el artículo denominado Orientación general sobre las anomalías mentales, como base del tratamiento pedagógico (Mann, 1912), aunque ya se había ocupado de esta temática unos años antes, incluso en sus primeras publicaciones, de corte más bien teórico (Mann, 1905b, 1909, 1910).

Ya en su Memoria sobre la Instalación del Laboratorio de Psicolojia Esperimental (Mann, 1908), se ocupa de varios casos en los que la aplicación de la metodología experimental se articula con el problema de la anormalidad infantil en Hungría, Austria y Bélgica, además de abordar el trabajo realizado en laboratorio instalado en el Asilo de Villejuif en Francia, para el diagnóstico del estado mental de los pacientes y "la determinación exacta de cualquier individualidad" (Mann, 1908, p. 303) a cargo de Toulouse y Piéron. Además de esta, se destacan las investigaciones realizadas por Alfred Binet en el Laboratorio de Psicología de la Sorbona y el Laboratorio de Pedagogía Normal que, como se comentó previamente, permitieron elaborar un procedimiento para el diagnóstico y reubicación de los niños anormales.

Específicamente, el cuadro que presentaba el panorama francés impulsó a Mann a una breve pero contundente conclusión sobre el estatuto de lo anormal en el marco de las indagaciones psicopedagógicas: "[d]ondequiera que se haya aplicado en una escala más vasta la esperimentacion psicolójica a las cuestiones de educación, los investigadores se han visto conducidos a la presencia de fenómenos psíquicos anormales" (Mann, 1908, p. 305).

En el caso de Francia, destaca la labor de la Sociedad Libre de Estudio Psicológico del Niño, que cuenta con diversas comisiones dedicadas al estudio de problemas psicológicos específicos y difunde sus investigaciones mediante su boletín. Pero, de manera más concreta, Mann atrae la atención sobre el particular interés de los franceses por "la investigación de los estados normales de conciencia" (Mann, 1908, p. 306) y por el uso del hipnotismo (y la sugestión) como método experimental y terapéutico por parte de Charcot, Ribot y la Escuela de Nancy. A partir de esto, planteó, como una de las tareas a realizar en el laboratorio local, poner a prueba las hipótesis que proponen "la posibilidad i necesidad de curar ciertas perturbaciones del ser intelectual o del ser moral del niño, mediante sujestiones proporcionadas a él en el estado hipnotizado" (Mann, 1908, p. 306). Con este fin, contempló incluso adquirir algunos instrumentos, como, por ejemplo, un aparato de hipnotización de Verdín.

En este mismo artículo, al dar cuenta de las características generales de los estudios psicológicos experimentales, se ocupó de la aplicación de la experimentación psicológica en relación con la pedagogía, la cual habría dado lugar a la pedagogía experimental (Mann, 1908); disciplina que se ocupa de estudiar al educador y al educando y, en el caso de este último, tanto en sus aspectos normales como particulares e individuales.

Estos estudios servirán de base, según Mann, a una orientación totalmente novedosa de la práctica educativa, complementaria de las actuales actividades escolares, y que permitirían organizar "ejercicios formales de las aptitudes elementales de la conciencia" (Mann, 1908, p. 330), e implementar una educación renovada que impactaría de manera beneficiosa a los niños anormales. De hecho, Mann afirmaba que ya se habían creados cursos auxiliares o de perfeccionamiento para estos casos.

Naturalmente, no se trata aquí de idiotas ni de imbéciles, sino de aquellos que pueden llegar a ser miembros útiles de la sociedad: pero que requieren para ello una educación especial, correctiva. Niños de esta categoría se encuentran casi sin escepcion en cada colejio, donde impiden al profesor de progresar con la rapidez que corresponde al término medio de los alumnos i donde al fin, a pesar de todos sus esfuerzos, ellos mismos fracasan. Estos niños deficientes o tardíos deben ser sometidos a un diagnóstico esperimental, por el cual se constatan los factores que causan su atraso mental (Mann, 1908, p. 331). 
Estos últimos aspectos, junto con las propuestas formuladas por Mann sobre el impacto de la doctrina spenceriana en el ámbito de la pedagogía (Mann, 1905b), son retomadas poco después en un artículo que sale a luz bajo el título $E l$ cultivo de la individualidad en la enseñanza escolar (Mann, 1910), donde se ocupa de dos tipos particulares y diferentes de individualidades: los alumnos sobrenormales y los niños con aptitudes reducidas, cada uno de las cuales requiere abordajes diferentes. En efecto, mientras rechazaba la idea de crear establecimientos especiales para estudiantes con talentos extraordinarios, planteaba las dificultades que genera la admisión de los débiles mentales en los colegios públicos.

De esta forma, se ocupó de exponer las particulares características de las clases auxiliares o de perfeccionamiento instalados por el Dr. Joseph Anton Sickinger en la ciudad de Mannheim, Alemania, a partir de 1905, y al presentar con bastante detalle sus divisiones y articulaciones, se interesó por mostrar que este sistema permitía una mayor individualización de la práctica educativa, a la vez que apreciaba el valor de "los principios que establece la ciencia de la economía de las enerjías psíquicas" (Mann, 1910, p. 311) para disponer, desde una perspectiva higiénica, tanto los horarios, la duración y el espacio para las clases, como la alimentación de los estudiantes.

Finalmente, como se mencionó anteriormente, en 1912 Wilhelm Mann publicó un artículo relativamente extenso para abordar la cuestión de las anomalías mentales y el papel de la pedagogía en su tratamiento. Allí, retomaba la matriz spenceriana, palpable desde muy temprano en su obra (Mann, 1905b), y respondía a las eventuales objeciones que se levantaron contra la educación de los niños mentalmente anormales: por una parte, decía, la educación puede elevar su nivel y transformarlos en elementos positivos de la colectividad; $y$, por otra, aunque la educación y la beneficencia se despreocuparan de ellos, encontrarían igualmente el modo de preservarse y obrar de ese modo como elemento perturbador de la sociedad: "[p]rueba de esto es el hecho de que la mayor parte del núcleo principal de los criminales provienen de su contingente de individuos anormales" (Mann, 1912, p. 596).

Dentro de ese "contingente", Mann consideró que los más interesantes son quienes ocupan un grado intermedio entre la normalidad y la anormalidad evidente o notoria, en tanto solían pasar inadvertidos y llegar incluso a ocupar puestos de autoridad en la sociedad, desde los cuales les resultaba posible ejercer una nefasta influencia. Una vía para evitar este panorama era instalar un sistema de selección en las escuelas, pero a Mann le parecía particularmente importante propagar el conocimiento sobre las anomalías mentales entre los educadores, y es con este fin que redactó este artículo.
Para ello, partiendo de la definición de las anomalías psíquicas o "defectos psico-patolójicos" como "fenómenos que están basados en deficiencias de constitución o funcionamiento nerviosos" (Mann, 1912, p. 599) proponía distinguir inicialmente dos clases: las perturbaciones mentales parciales -que no atacan a la personalidad entera o no lo hacen de manera permanente- y las psicopatías -que modifican mórbidamente la personalidad-. Pero, llegado a este punto, Mann incluyó a una variedad de grados intermedios entre la normalidad y anormalidad evidente dentro una tercera clase: los "estados subnormales" (Mann, 1912, p. 599).

Para esto, se apoyó en los desarrollos del psiquiatra alemán Julius Ludwig August Koch sobre la psychopathischen minderwertigkeiten [inferioridad psicopática], que definen como "desviaciones leves de la norma psíquica $\mathrm{sea}[\mathrm{n}]$ conjénitas o adquiridas [...] como estados fronterizos del gran dominio de las neurosis [...] sobre todo como formas leves de la inestabilidad, astenia e histeria [...] como formas de lijera debilidad mental" (Mann, 1912, pp. 599-600). Por lo general, esta inferioridad, señala Mann, está caracterizada por una excesiva ingenuidad, por lo que quienes la presentan no logran entender las situaciones en las que se hallan y no están en condiciones de adaptarse a ellas: sus comportamientos suelen mostrar características claramente infantiles.

La mayor parte del artículo está dedicado al segundo de los tres grupos enumerados por Mann: las psicopatías, que incluyen a la psicosis -cuya sintomatología es principalmente mental-, las neurosis - con una sintomatología predominantemente física- y la degeneración moral -categoría polémica, atravesada por los debates sobre las relaciones entre intelecto y moral-. En el caso de las psicosis, distingue entre orgánicas -defectos de inteligencia- y funcionales -en las que la merma intelectual no es notoria, como la melancolía pura, la locura maníaca-depresiva y la paranoia-. Si bien su tratamiento es de incumbencia médica, Mann planteó la posibilidad de intervención de la escuela auxiliar de niños anormales, sobre todo en aquellos casos que conducen a la debilidad mental, como la demencia.

El principal síntoma psicológico en las psicosis orgánicas es la deficiencia intelectual, ya sea congénita-detención del desarrollo cerebral-o adquirida -que no se presenta nunca antes de los diez años de edad, como la demencia-. Esta última consiste más bien en una regresión de las facultades mentales y un "empobrecimiento progresivo del contenido de la conciencia i de la capacidad de las funciones mentales" (Mann, 1908, p. 408), y Mann se detiene en la forma hebefrénica de la demencia precoz, que no debe ser confundida por el educador con un sujeto perezoso o descuidado.

Asimismo, Mann distingue, dentro de la psicosis orgánica, tres grados de debilidad mental: la idiotez-caso extremo de 
imposible educación-, la imbecilidad y la debilidad ligera, a lo que habría que agregar la diferenciación entre aquellos casos de debilidad mental por causas centrales -en los que resulta prácticamente imposible atacar la causa directa- $\mathrm{y}$ por causas no centrales. Entre estas últimas, Mann menciona a las dificultades respiratorias por vegetaciones adenoides y algunas enfermedades infecciosas - escarlatina, difteria, sarampión o tifus-, cuya curación, mediante tratamiento médico, deja espacio para los aportes de una escuela auxiliar que se adapte a este tipo de casos.

Asimismo, Mann menciona algunos casos de debilidad mental aparente, que con frecuencia consisten en anomalías de la visión o de la audición que pueden confundirse con debilidad mental o falta de voluntad; y en otros casos, se puede tratar de atrasados pedagógicos, personas que pueden poseer excelentes aptitudes mentales, pero que no las han ejercitado.

Posteriormente, Mann pasa a considerar el caso de las neurosis, empezando por el caso de la neurastenia, en sus dos formas: inestables -el indisciplinado escolar, que posee una remarcada excitabilidad nerviosa- y asténicos -solo capaces de tareas muy acotadas debido a la debilidad de su sistema nervioso-; y menciona que casos en los que se presentan transiciones de una a otra de estas dos formas, se debería aplicar "un tratamiento educativo especial" (Mann, 1912, p. 419) que considere las particularidades de sus energías psíquicas.

Tras considerar estas formas de neurastenia, el autor aborda el caso de la histeria, que, según el artículo, se presenta bajo apariencias muy diversas: alteraciones del movimiento o de la sensación, emocionalidad excesiva e inestable, o un rol demasiado preponderante del yo en sus intereses y conductas, que son de tratamiento exclusivamente médico, excepto en los casos en que los síntomas histéricos se concentran en la inteligencia, ya que es posible una intervención educativa de tipo correctivo en virtud de sus particulares características:

En la escuela ordinaria, estos niños histéricos sufren por impresiones i exigencias que están por encima de sus fuerzas nerviosas i además son un peligro para los demás, así como lo prueban las epidemias de histeria que por obra del contajio se han apoderado de cursos escolares enteros (Mann, 1912, p. 421).

En el cierre de este texto, además de resumir el contenido del artículo, Mann hace referencia a los dispositivos pedagógicos que comenzaron a instalarse especialmente para el caso de los débiles mentales y para los niños neuróticos. En este sentido, planteó que la educación especial de niños anormales "es capaz de conservar i desarrollar su pobre caudal i representa así una obra de la más alta importancia social" (Mann, 1912, p. 424) ${ }^{1}$.

Sin embargo, más allá de la precisión y de las propuestas planteadas por Mann, este panorama no se corresponde con lo que en esos años se estaba realizando a nivel local en el ámbito de la educación. Si bien se habían establecido hace tiempo espacios de encierro para casos psiquiátricos, no existían todavía dispositivos que se encargaran de las anomalías infantiles y tuvo que esperarse al menos una década para que se dispusieran espacios y prácticas para la detección y el tratamiento de los débiles mentales. Solo fue hasta mediados de la década de 1920 que tanto desde los saberes de la psicología como desde la administración estatal los residuos de la disciplina escolar se transformaron en un amplio foco de preocupación en Chile.

\section{Discusión}

En efecto, en lo que se refiere a las disposiciones reglamentarias, fue necesario esperar las reformas educativas que tuvieron lugar unos años más tarde para que se proyectaran una serie de instituciones que se ocuparon de la infancia anormal. Si bien ya se había promulgado en 1912 una ley de protección a la infancia desvalida, a través de ella solo se buscaba resolver cuestiones como el abandono paterno, el abuso de menores y algunas formas de explotación laboral. Lo mismo puede plantearse respecto del Primer Congreso Nacional de Protección a la Infancia que se realizó ese mismo año (Vial, 1912).

Ocho años después, en 1920, se sancionó la Ley de Educación Primaria Obligatoria(Dirección Jeneral deEducación Primaria, 1920), que reemplazó la legislación que había sido promulgada en 1860, y que lograba resolver una larga serie de debates que surgieron, entre otras cuestiones, respecto de la imposición de la obligatoriedad de la educación escolar, que ya se había implementado en varios países europeos e, incluso, latinoamericanos. Sin embargo, esa legislación nada indica respecto de la situación de aquellos que -por diversos motivos- pudieran ser irradiados de dispositivo escolar.

Muy poco tiempo después que esta ley fue promulgada se volvieron notorias las deficiencias con respecto a que no logró imponer la obligatoriedad y masividad a las que

\footnotetext{
No deja de resultar llamativo que, en posteriores publicaciones, Mann casi no vuelve a ocuparse del tema de la infancia anormal. De hecho, en una de sus escasas posteriores referencias a la psicopatología planteaba que el "estudio sistemático de las diferentes clases de anomalías mentales" por parte de los estudiantes no solamente carece de valor práctico, sino que puede aun ejercer una influencia desfavorable sobre el desarrollo tranquilo de su espíritu (Mann, 1915b).
} 
aspiraba, pero logró impulsar una reforma educacional que comenzó a implementarse hacia el final de la década de 1920. Entre sus innovaciones se destacan aquellas referidas a la educación de los sujetos con algún tipo de deficiencia mental que, hasta entonces, "debían resignarse a no recibir educación y, en algunos casos, a vivir encerrados en sus casas" (Rojas-Flores, 2010, p. 344). En efecto, el decreto con fuerza de ley (DFL) n ${ }^{0} 5291$ o Ley de Enseñanza Primaria Obligatoria, promulgado el 22 de noviembre de 1929, permitió apreciar claramente estas novedades: en ella se mencionan, además de las escuelas para ciegos y sordomudos -que ya existían hace varias décadas-, los establecimientos para débiles mentales, a los que se consideró como Escuelas de Experimentación Amplia. Además, esta nueva ley estipuló el establecimiento de una Escuela Experimental de Desarrollo en Santiago.

Sin embargo, las nuevas instituciones que fueron creadas resultaron insuficientes para producir un cambio sustancial en el destino de muchos de estos niños, a lo cual se agrega que "[t]ampoco hubo tratamiento para las alteraciones más moderadas" (Rojas-Flores, 2010, p. 344). En este último caso, fue necesario esperar hasta mediados de la década siguiente, cuando se creó la Clínica de Conducta con el auspicio de Claudio Matte Pérez, presidente de la Sociedad de Instrucción Primaria (Agüero-Correa, 1938).

En el ámbito más estrictamente académico-profesional, unos de los sucesores de Wilhelm Mann fue quien se ocupó en difundir ciertas técnicas que permitían intervenir en el marco de la anormalidad infantil: Luis Tirapegui, doctorado en la Universidad de Columbia, se hizo cargo del Laboratorio Experimental del Instituto Pedagógico en el año 1923 y se ocupó de adaptar al panorama chileno los principales test de inteligencia. En 1925, Tirapegui publicó El desarrollo de la intelijencia medido por el método Binet-Simon (Tirapegui, 1925), documento en el que se menciona por primera vez la revisión de la escala de inteligencia francesa llevada a cabo en Stanford por Lewis Terman, con base en la cual Tirapegui propuso su implementación local.

Este proyecto parece haber resonado en el marco de las políticas que comenzaba a implementar el Estado chileno, por lo que la adaptación del test realizada por Tirapegui fue editada y distribuida por el Ministerio de Educación Pública, el cual impuso su aplicación sobre los niños chilenos durante varias décadas (Sánchez, 2014).

Para que lograra imponerse una "función psi" en Chile, no bastaba entonces con los desarrollos teóricos ni con las instituciones que hace décadas se habían dispuesto para abordar el caso de los niños anormales en Europa y que bastante temprano comenzaban a difundirse en Chile por Wilhelm Mann. Fue necesario que el contexto local habilitara e incentivara, a la luz de los problemas que fue planteando la instalación del dispositivo escolar, la búsqueda de las herramientas que permitieran solucionarlos por parte de las autoridades gubernamentales. En este marco, según algunas investigaciones recientes (Leyton, Palacios \& Sánchez, 2015; Sánchez, 2014), el problema de la inteligencia se articuló con el paulatino despliegue de la eugenesia en Chile.

En conclusión, el análisis de Foucault sobre la escuela como institución normalizada fue permitiendo tanto al Estado como a la escuela establecer diferencias significativas respecto a la concepción de la infancia, debido a que se establecieron parámetros de normalidad y anormalidad respaldados por los saberes "psi" que generaron distinciones y segregaciones que contribuyeron finalmente tanto a la formación docente especializada como a la creación de centros particulares donde los desadaptados o anormales debieron ser recluidos, segregados y categorizados, según sus capacidades. Mann, en un afán normalizador, logró homogeneizar el proceso educativo, que de la mano de los planteamientos de autores como Binet y Simon, entre otros, establecieron los cimientos centrales del sistema educativo chileno y, con ello, las definiciones de infancia.

\section{Referencias}

Agüero-Correa, G. (1938). Psiquiatría e higiene mental infantil, nuestra experiencia. Acción Social, VII(66), 31-39.

Barrera, (2010). Orígenes de la psicología educacional chilena: 1885-1919. En M. A. Laborda \& V. E. Quezada (eds.), Notas historicas de la psicologia en Chile (pp. 163-191). Santiago: Editorial Universitaria.

Binet, A. (1906). Introducción a la psicología experimental. Madrid: Daniel Jorró Editor.

Binet, A., \& Simon, T. (1904). Méthodes nouvelles pour le diagnostic du niveau intellectuel des anormaux. L'Année Psychologique, 11, 191-244. doi: https://doi.org/10.3406/ psy. 1904.3675

Binet, A., \& Simon, T. (1907). Les enfants anormaux. Guide por l'admission des Enfants anormaux dans les clases de Perfectionnement. Paris: Colin.

Binet, A., \& Simon, T. (1908). L'intelligence des imbéciles. L'Année Psychologique, 15, 1-147. doi: https://doi. org/10.3406/psy.1908.3754

Caiceo, J. (2011). Pensamiento pedagógico en Chile en el siglo XX. Desde la escuela nueva al constructivismo. Teoria e Prática da Educação, 14(2), 7-20. doi: 10.0000/rtpe. v14i2.16148

Campos, F. (1960). Desarrollo educacional. 1810-1960. Santiago: Editorial Andrés Bello.

Carr, E.(1993). ¿Quées la historia? Barcelona:Planeta-Agostini.

Ciudad, M. (1989). El Instituto Pedagógico y la educación laica. Santiago: Universitaria. 
Cox, C., \& Gysling, C. (2009). La formación del profesorado en Chile. 1842-1987. Santiago: Ediciones Universidad Diego Portales.

De la Barra, E. (1899). El embrujamiento alemán. Santiago: Establecimiento Poligráfico Roma.

De Mussy, L., \& Valderrama, M. (2010). Historiografía postmoderna. Conceptos, figuras, manifiestos. Santiago: Ril Editores.

Dirección Jeneral de Educación Primaria. (1920). Lei $N^{o} 3,654$ sobre Educación Primaria Obligatoria. Santiago: Imprenta Lagunas \& Co.

Egaña, M. L. (2000). La educación primaria popular en el siglo XIX en Chile: una práctica de política estatal. Santiago: LOM.

Foucault, M. (1990). Las tecnologías del yo. Barcelona: Paidós.

Foucault, M. (1999). Estrategias de Poder. Barcelona: Paidós.

Foucault, M. (2001). Los anormales. Curso en el College de France (1974-1975). Buenos Aires: FCE. (Trabajo original publicado en 1999).

Foucault, M. (2005). El poder psiquiátrico. Curso en el College de France (1973-1974). Buenos Aires: FCE. (Trabajo original publicado en 2003).

Foucault, M. (2014a). Historia de la sexualidad volúmen 1. Buenos Aires: Siglo XXI.

Foucault, M. (2014b). Defender la sociedad. Argentina: FCE.

Foucault, M. (2016). La sociedad punitiva. Buenos Aires: FCE.

Foucault, M. (2017). La verdad y las formas jurídicas. Barcelona: Gedisa.

Fuentealba, L. (1964). Valentín Letelier y el pensamiento educativo en la época de fundación del Instituto Pedagógico. Santiago: Universitaria.

García, J. E. (2016). La introducción de la Escala de Inteligencia Stanford-Binet en el Paraguay. Interacciones, 2(1), 65-83. doi: 10.24016/2016.v2n1.28

García-Yagüe, J. (2010). Entre la euforia y el desaliento. Las grandes aportaciones de Binet y su impacto en el desarrollo psicopedagógico del siglo XX. Tendencia Pedagógicas, 15(1), 1-28. Recuperado de https://revistas.uam.es/ tendenciaspedagogicas/article/view/1931

Goody, J. (2009). La evolución de la familia y el matrimonio. Valencia: Universitat de València.

Huertas, R. (2008). Los laboratorios de la norma: medicina y regulación social en el estado liberal. Barcelona: Octaedro.

Huertas, R. (2011). En torno a la construcción local de la locura. Ian Kacking y la historia cultural de la psiquiatría. Revista de la Asociación Española de Neuropsiquiatría, 31(311), 413-437. doi: 10.4321/S0211-57352011000300004

Huertas, R. (2014). ¿Qué sabemos de? La locura. Madrid: Catarata.

Huertas, R., \& del Cura, M. (1996). La categoría "infancia anormal" en la construcción de una taxonomía social. Asclepio, 48(2), 115-127. doi: https://doi.org/10.3989/asclepio.1996. v48.i2.400
Illanes, M. A. (1991). Ausente, señorita: el niño chileno, la escuela para pobres y el auxilio: 1890/1990: (hacia una historia social del siglo XX en Chile). Santiago: JUNAEB.

Kant, I. (1764/2018). Ensayo sobre las enfermedades de la cabeza. Madrid: Antonio Machado.

Klappenbach, H. (2014). Acerca de la metodología de la investigación en historia de la psicología. Psykhe, 23(1), 1-12. doi: http://dx.doi.org/10.7764/psykhe.23.1.584

Labarca, A. (1939). Historia de la enseñanza en Chile. Santiago: Universitaria.

Lapoujade, M. N. (2015). El imprescindible ensayo de las enfermedades de la cabeza de Kant (1764). Ciencia Latinoamericana, 9(1), 107-113. doi: https://doi. org/10.22235/cp.v9i1.170

Larraín, J. I., \& Larraín, J. J. (1871). Boletín de las principales leyes y decretos dictados desde Enero 1 de 1860 hasta Enero 1 de 1871. Valparaíso: Imprenta del Mercurio De Tornero y Letelier.

Larrosa, J. (1995). Escuela, poder y subjetivación. Madrid: La Piqueta.

Letelier, V. (1940). El instituto pedagógico. Misceláneas de estudios pedagógicos. Santiago: Instituto Cultural Germano Chileno.

Leyton, C., Palacios, C., \& Sánchez, M. (2015). El bulevar de los pobres. Racismo cientifico, higiene y eugenesia, siglos $X I X$ y XX. Santiago: Editorial Ocho Libros.

Lieberman, J. A. (2016). Historia de la psiquiatría. De sus orígenes, sus fracasos y su resurgimiento. Barcelona: Ediciones B.

Mann, W. (1905a). Jorge Enrique Schneider, su acción en el progreso de la Filosofía. Anales de la Universidad de Chile, 117, 1-17. doi: 10.5354/0717-8883.2012.24632

Mann, W. (1905b). La doctrina de Herbert Spencer i las directrices para el progreso de la educación. Anales de la Universidad de Chile, 116, 519-573. doi:10.5354/0717-8883.2012.24256

Mann, W. (1906). Lecciones introductorias la pedagojia esperimental. Anales de la Universidad de Chile, 123, 226-321. doi: 10.5354/0717-8883.2012.24741

Mann, W. (1908). Memoria sobre la Instalación del Laboratorio de Psicología Experimental. Anales de la Universidad de Chile, 123, 279-340. doi: 10.5354/0717-8883.2012.24266

Mann, W. (1909). La organización del laboratorio chileno de psicolojía esperimental. Anales de la Universidad de Chile, 124, 665-688. doi: 10.5354/0717-8883.2012.24115

Mann, W. (1910). El cultivo de la individualidad en la enseñanza escolar. Anales de la Universidad de Chile, 127, 289320. doi: 10.5354/0717-8883.2012.25058

Mann, W. (1912). Orientación jeneral sobre las anomalías mentales como base de su tratamiento pedagójico. Anales de la Universidad de Chile, 130, 593-626. doi: 10.5354/0717-8883.2012.25469 
Mann, W. (1915a). El espíritu jeneral i los ramos de enseñanza filosófica en el Liceo. Anales de la Universidad de Chile, 73, 643-707. doi:10.5354/0717-8883.2012.24295

Mann, W. (1915b). La enseñanza de la psicolojía i de la lójica en el Liceo. Anales de la Universidad de Chile, 73, 939976. doi:10.5354/0717-8883.2012.24302

Mellafe, R. (1988). Reseña histórica del Instituto Pedagógico: cien años en la formación profesores. Santiago: Universidad Metropolitana de Ciencias de la Educación.

Mellafe, R., \& González, M. T. (2007). El Instituto Pedagógico de la Universidad de Chile. (1889-1981): su aporte a la educación, cultura e identidad nacional. Santiago: LOM.

Ministerio de Justicia, Culto e Instrucción Primaria (1833). Reglamento para la enseñanza i régimen interno de las escuelas elementales. En Boletín de leyes i decretos del gobierno (p. 349). Santiago: Imprenta Nacional.

Muel-Dreyfus, F. (1975). L'école obligatoire et l'invention de l'enfance anormale. Actes de la Recherche en Sciences Sociales, 1(1), 60-74. doi: https://doi.org/10.3406/ arss. 1975.2450

Muñoz, J. M. (1918). Historia elemental de la pedagojia chilena. Santiago: Sociedad Imprenta y Litografía Universo.

Noll, S., \& Trent, J. (eds.) (2004). Mental retardation in America: a historical reader. New York: New York University Press.

Núñez, J. A. (1883). Organización de escuelas normales. Santiago: Imprenta de la Librería Americana.

Núñez, J. A. (1890). Congreso pedagójico. Resúmen de las discusiones, actas i memorias presentadas al primer congreso pedagojico celebrado en Santiago de Chile. Santiago: Imprenta Nacional.

Parra, D. (2015). Un análisis historiográfico sobre las relaciones entre psicología y educación en Chile. 1889-1973. Revista de Historia de la Psicología, 36(2), 95-114. Recuperado de https://www.revistahistoriapsicologia.es/ app/download/6022482711/5\%20PARRA\%20MORENO. pdf?t=1433431974

Postel, J., \& Quétel, C. (coord.). (2000). Nueva historia de la psiquiatría. México: Fondo de Cultura Económica.

Ramírez, C., \& Navarrete, G. (1932). Rómulo. J. Peña Maturana. Revista Pórtico. Publicación Extraordinaria Atacama. 127. Copiapó: Talleres Progreso.

Ravier, J. (2012). L'Evaluation des enseignants du primaire en France de 1835 à 1850. Spirale - Revue de Recherches en Éducation, 49, 37-51. doi: https://doi.org/10.3406/ spira.2012.1107

Rosa, A., Huertas, J. A., \& Blanco, F. (1996). Metodología para la historia de la psicología. Madrid: Alianza.

Rojas-Flores, J. (2010). Historia de la infancia en el Chile republicano, 1810-2010. Santiago: JUNJI.

Salas, G. (2012). La influencia europea en los inicios de la historia de la psicología en Chile. Interamerican Journal of Psychology, 46(1), 99-110. doi: https://doi.org/10.30849/ rip/ijp.v46i1.185
Salas, G., \& Lizama, E. (2013). Historia de la psicología en Chile. 1889-1981 (2. ${ }^{a}$ ed). La Serena: Universidad de La Serena.

Salas, G., Scholten, H., \& Rey-Anacona, C. (2015). Nadie nace civilizado. En S. Saavedra, G. Salas, C. Cornejo \& P. Morales (eds.), Resiliencia y calidad de vida. La psicología educacional en diálogo con otras disciplinas (pp. 61-73). Talca: UCM.

Salas, G., Norambuena, Y., Scholten, H., \& Torres-Fernández, I. (2018). Psicología y educación en Chile. Problemas, perspectivas y vías de investigación. 1860-1930. Universitas Psychologica. 17(5), 1-14. doi: 10.11144/Javeriana.upsy175.pecp

Sánchez, J. J., \& Valderrama-Iturbe, P. (2001). Psychology in Latin America: historical reflections and perspectives. International Journal of Psychology, 36(6), 384-294. doi: https://doi.org/10.1080/00207590143000216

Sánchez, M. (16 de septiembre, 2014). La sabiduría de los fabricantes de sombreros. La inteligencia en la eugenesia chilena. Recuperado de http://nuevomundo.revues.org/67197

Sarmiento, D. F. (1896a). De la educación popular. Buenos Aires: Imprenta y Litografía Mariano Moreno.

Sarmiento, D. F. (1896b). Civilización o barbarie. Buenos Aires: Imprenta y Litografía Mariano Moreno.

Seguin, E. (1846). Traitement moral, hygiène et éducation des idiots et des autres enfants arriérés. Paris: Chez J. B. Baillière.

Serrano, S., Ponce de León, M., \& Rengifo, F. (2012). Historia de la educación en Chile (1810-2010) Tomo II, La educación nacional (1980-1930). Santiago: Taurus.

Smajda, E. (2013). La pareja y su historia. Buenos Aires: Biblos.

Tedesco, J. C., \& Zacarías, I. (2011). Domingo Faustino Sarmiento. La vigencia de su pensamiento educativo. En D. F. Sarmiento (ed.), Educación popular (pp. 9-25). Buenos Aires: UNIPE.

Terigi, F. (2009). El fracaso escolar desde la perspectiva psicoeducativa: hacia una reconceptualización situacional. Revista Iberoamericana de Educación, 50, 23-29. Recuperado de https://rieoei.org/RIE/article/view/659

Tirapegui,L. (1925). El desarrollo de la inteligencia medida porel método Binet-Simon. Anales de Psicología, Segunda Serie, Tomo III, 453-591. doi: 10.5354/0717-8883.2012.25591

Urzúa, A., Vera-Villarroel, P., Zúñiga, C., \& Salas, G. (2015). Psicología en Chile: análisis de su historia, presente y futuro. Universitas Psychologica, 14(3), 1125-1142. doi: 10.11144/Javeriana.upsy14-3.pcah

Vial, M. C. (1912). Trabajos y actas del Primer Congreso Nacional de Protección a la Infancia. Santiago: Impr. Barcelona.

Voisin, F. (1843). De L'Idiotie chez les enfants et des autres particularités d'intelligence ou de caractére qui nécessitent pour eux une instructon et une éducation spéciales de leur responsabilité morale. París: Chez J. B. Baillière. 